報 文 3

\title{
Visible Emission Systems in Silicon Monoiodide
}

\author{
Masaharu TsujI, Keiji Shinohara,* and Yukio Nishimura \\ Research Institute of Industrial Science, and Department of Molecular Science \\ and Technology, Graduate School of Engineering Sciences, Kyushu University, \\ Kasuga-shi. Fukuoka 816
}

(Received Septemqer 29, 1983)

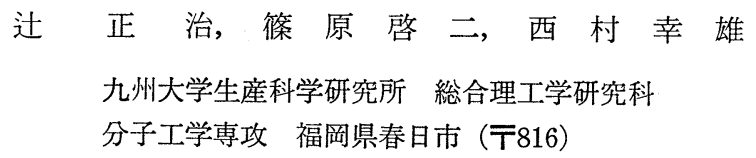

(1983年 9 月 29 日受理)

\begin{abstract}
The optical spectroscopic study of the Ar afterglow reaction of $\mathrm{SiI}_{4}$ has led to the observation of two visible emission systems of SiI. A new red-degraded emission in the $430-480$ $\mathrm{nm}$ region was assigned to the $A^{2} \Sigma^{+}-X^{2} \Pi_{1 / 2}$ system by comparison with absorption spectrum. The $a\left({ }^{4} \Sigma^{-}{ }_{1 / 2}\right)-X^{2} \Pi_{3 / 2}$ system was detected in the $490-560 \mathrm{~nm}$ region. The intensity was so strong that several new bands could be identified in longer wavelengths. The excitation source of SiI emission was concluded to be metastable $\mathrm{Ar}^{+}$ions.
\end{abstract}

\section{Introduction}

Although ultraviolet and visible band systems of SiI have been well known in absorption, only one system has been detected in emission. Lakshminarayana and Haranath" first discovered a SiI emission in the 480-550 $\mathrm{nm}$ region excited by a radio frequency discharge through gaseous $\mathrm{I}_{2}$ over silicon metal. The spectrum was composed of two subsystems; a weaker subsystem was located at $650 \mathrm{~cm}^{-1}$ to the red of the main subsystem. They have attributed this doublet system to the $A^{2} \Sigma^{+}-$ $X^{2} \Pi_{r}$ transition by analogy with previously known systems of silicon monohalides. Billingsley ${ }^{2)}$ re-examined the main subsystem under a higher resolution and re-assigned it to the
$A^{\prime}-X^{2} \Pi_{3 / 2}$ transition. He suggested that the $A^{\prime}$ state is possibly the case $\left(a^{\prime}\right)^{4} \Sigma_{1 / 2}^{-}$state which interacts with the $A^{2} \Sigma^{+}$state. Therefore, Huber and Herzberg. ${ }^{3)}$ have listed the $A^{\prime}$ state as tentatively the $a^{4} \Sigma_{1 / 2}^{-}$state. Billingsley ${ }^{2)}$ reported that the weaker subsystem could not be observed in his spectrum obtained from a microwave discharge through $\mathrm{Si}$ and $\mathrm{I}_{2}$.

We have studied emission spectra produced from the Ar afterglow reaction of $\mathrm{SiI}_{4}$. Two visible emission systems of Sil have been observed and analyzed vibrationally. They are attributed to the $A^{2} \Sigma^{+}-X^{2} \Pi_{1 / 2}$ and $a\left({ }^{4} \Sigma_{1 / 2}^{-}\right)$ $-X^{2} \Pi_{3 / 2}$ transitions by comparison with reported absorption and emission spectra. The former emission system is detected for the first time in this work.

* Now at SONY Corporation, Atsugi, Kanagawa 243. 


\section{Experimental}

The flowing afterglow apparatus used has been described previously ${ }^{4}$. Emission spectra produced from reactions of active $\mathrm{Ar}$ species with $\mathrm{SiI}_{4}$ (CERAC Chem. Co.) were measured in the $200-700 \mathrm{~nm}$ region by using, a Jarrell Ash $1 \mathrm{~m}$ monochromator. The pressure of $\mathrm{Ar}$ was $0.2-0.6$ Torr. In order to obtain a sufficiently high pressure of $\mathrm{SiI}_{4}$ in the reaction zone, it was necessary to heat the sample at about $370 \mathrm{~K}$. Available atomic lines in the flame spectra were used as wavelength standards. The error in the measurement of sharp bandheads was less than $0.05 \mathrm{~nm}$.

\section{Results and analysis}

Emission spectrum of $\mathrm{SiI}_{4}$ in an Ar afterglow consists of sharp lines of Si and I atoms and characteristic SiI emissions in the visible region. Figure 1 shows a typical emission spectrum in the $430-560 \mathrm{~nm}$ region. Spectrum (a) was obtained by the $\mathrm{Ar}^{*}, \mathrm{Ar}^{+}$, and $\mathrm{Ar}^{+M}$
$+\mathrm{SiI}_{4}$ reactions, while spectrum (b) was detected after removing ionic species. Spectrum (a) is composed of intense atomic lines of iodine and two systems of SiI in the 430-480 and $490-560 \mathrm{~nm}$ region. The former system of SiI is a new emission observed in this work. A great suppression of emission intensity of SiI in spectrum (b) indicates that the dominant excitation source is ionic species, $\mathrm{Ar}^{+}$and/or $\mathrm{Ar}^{+M}$ (metastable ions). Because of lack of thermochemical data on $\mathrm{SiI}_{4}$ and SiI molecules, it is difficult to determine accurate minimum energies required for processes (1) and (2):

$$
\begin{aligned}
& \mathrm{SiI}_{4} \longrightarrow \mathrm{SiI}^{*}+\mathrm{I}_{2}^{+}+\mathrm{I}+e^{-} \\
& \mathrm{SiI}_{4} \longrightarrow \mathrm{SiI}^{*}+\mathrm{I}_{2}+\mathrm{I}^{+}+e^{-} .
\end{aligned}
$$

The sum of ionization potential of $\mathrm{I}_{2}(9.3 \mathrm{eV})$ or $\mathrm{I}(10.4 \mathrm{eV})$ and excitation energy of SiI (2.6$2.9 \mathrm{eV})$ is smaller than the recombination energies of $\mathrm{Ar}^{+}\left({ }^{2} \mathrm{P}_{3 / 2}: 15.76 \mathrm{eV}\right.$ and ${ }^{2} \mathrm{P}_{1 / 2}: 15.94$ $\mathrm{eV}$ ) by $2.5-4.0 \mathrm{eV}$. The minimum energy for dissociation process (3) is expected to be larger than $4.0 \mathrm{eV}$ on the basis of thermochemical data on similar processes $(4)-(6)^{5,6)}$ :
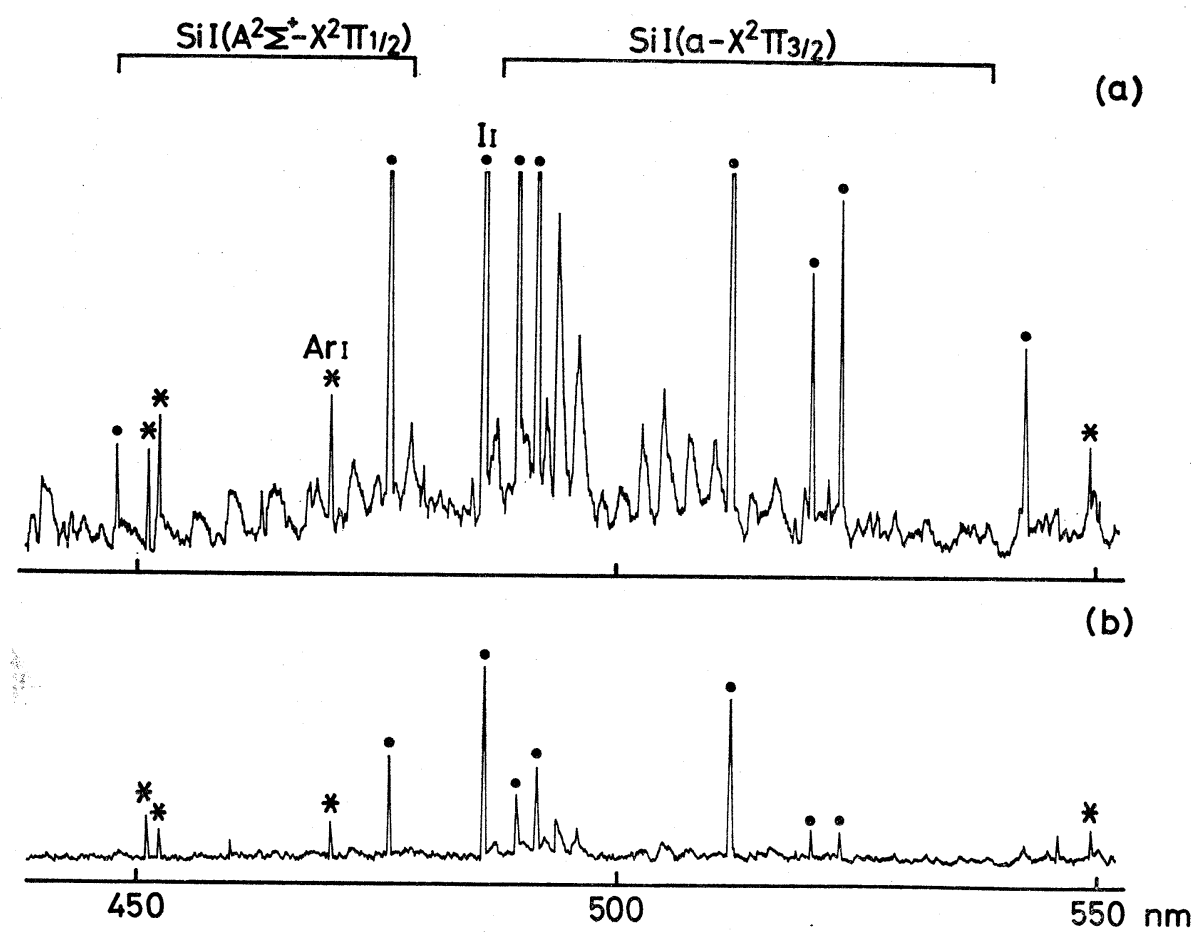

Fig. 1. Emission spectra produced from the (a) $\mathrm{Ar}^{*}, \mathrm{Ar}^{+}$and $\mathrm{Ar}^{+M}+\mathrm{SiI}_{4}$ and (b) $\mathrm{Ar}^{+}+\mathrm{SiI}_{4}$ reactions. Lines marked - and $*$ are $\mathrm{I}_{\mathrm{I}}$ and stray $\mathrm{Ar}_{\mathrm{I}}$ lines, respectivery. 


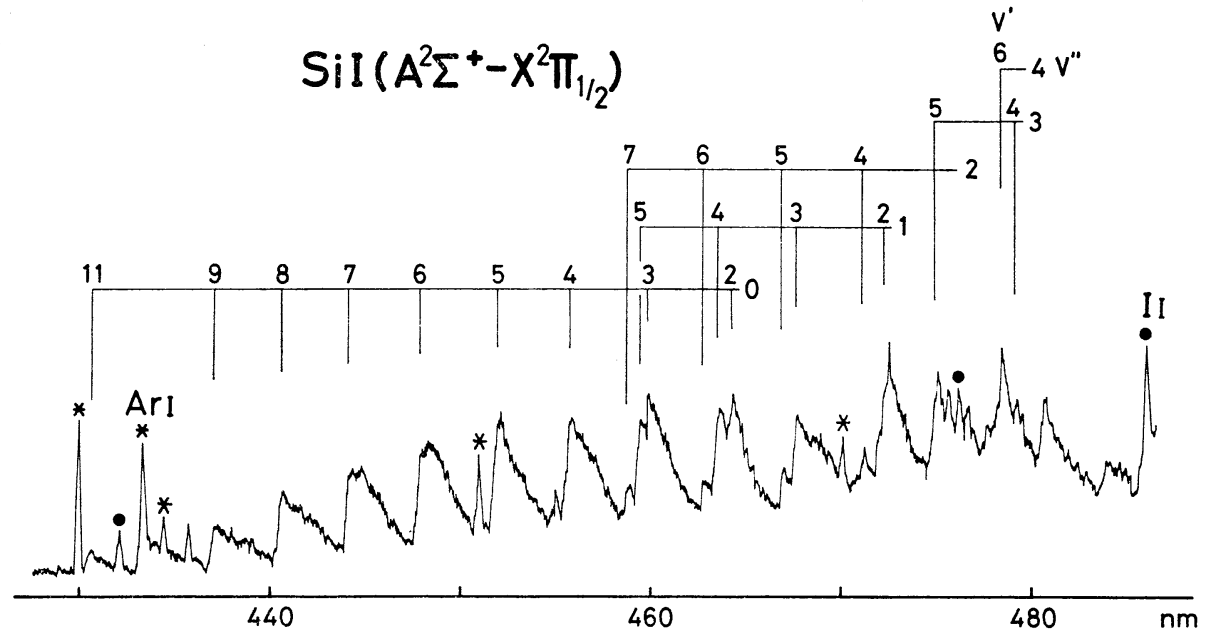

Fig. 2. New emission system of SiI in the $430-480 \mathrm{~nm}$ region.

$$
\begin{aligned}
& \mathrm{SiI}_{4} \longrightarrow \mathrm{SiI}+\mathrm{I}_{2}+\mathrm{I} \\
& \mathrm{SiF}_{4} \longrightarrow \mathrm{SiF}+\mathrm{F}_{2}+\mathrm{F}-17.4 \mathrm{eV} \\
& \mathrm{SiCl}_{4} \longrightarrow \mathrm{SiCl}+\mathrm{Cl}_{2}+\mathrm{Cl}-10.0 \mathrm{eV} \\
& \mathrm{SiBr}_{4} \longrightarrow \mathrm{SiBr}+\mathrm{Br}_{2}+\mathrm{Br}-8.0 \mathrm{eV}
\end{aligned}
$$

It was therefore concluded that the dominant excitation source was $\mathrm{Ar}^{+M}$ with available energies of $16.23-20.27 \mathrm{eV}$ for $\mathrm{Ar}^{+M}-\mathrm{Ar}^{+}$excitation transfer and of $17.87-22.18 \mathrm{eV}$ for $\mathrm{Ar}^{+M_{-}}$ $\mathrm{Ar}^{*}\left(3 \mathrm{p}^{5} 3 \mathrm{~d}\right)$ charge transfer ${ }^{7)}$.

In Fig. 2 is shown the SiI emission in the $430-480 \mathrm{~nm}$ region. This emission was absent in the $\mathrm{He}$ and $\mathrm{Ne}$ afterglow reactions of $\mathrm{SiI}_{4}$. The bands are single-headed and red-degraded. The observed wavelengths of bandhead and corresponding vacuum wavenumbers are listed in Table 1. The wavenumbers of dominant bandheads agree well with those of the $X \rightarrow A$ transition of SiI obtained from absorption spectrum $^{2}$. We therefore attributed the new emission to the $A^{2} \Sigma^{+}-X^{2} \Pi_{1 / 2}$ transition of SiI. In addition to $9\left(v^{\prime}, 0\right)$ bands identified in absorption, 11 new bands could be detected in this work. The observed wavenumbers are compared with those predicted from reported molecular constants. The calculated wavenumbers are reproduced the experimental observation within $6 \mathrm{~cm}^{-1}$, indicating the validity of our vibrational assignment. The absence of emission from low $v^{\prime}$ levels such as $v^{\prime}=0$ and 1 and the observation of intense $v^{\prime \prime}$ $=0 v^{\prime}$-progression imply that internuclear sepa-

\begin{tabular}{|c|c|c|c|c|}
\hline \multirow{2}{*}{$v^{\prime}$} & \multirow{2}{*}{$v^{\prime \prime}$} & \multirow{2}{*}{$\begin{array}{l}\text { Wavelength } \\
(\AA)\end{array}$} & \multicolumn{2}{|c|}{ Wavenumber $\left(\mathrm{cm}^{-1}\right)$} \\
\hline & & & Observed & Obs.-Cal.* \\
\hline 11 & 0 & 4304.7 & 23224 & -3 \\
\hline 9 & 0 & 4369.8 & 22878 & 0 \\
\hline 8 & 0 & 4405.0 & 22695 & -1 \\
\hline 7 & 0 & 4440.8 & 22512 & 2 \\
\hline 6 & 0 & 4478.3 & 22324 & 3 \\
\hline 5 & 0 & 4518.0 & 22128 & -1 \\
\hline 4 & 0 & 4557.0 & 21938 & 5 \\
\hline 7 & 2 & 4587.6 & 21792 & 2 \\
\hline 5 & 1 & 4593.9 & 21762 & -5 \\
\hline 3 & 0 & 4599.3 & 21736 & 0 \\
\hline 6 & 2 & 4628.1 & 21601 & 0 \\
\hline 4 & 1 & 4634.8 & 21570 & -2 \\
\hline 2 & 0 & 4642.2 & 21535 & 0 \\
\hline 5 & 2 & 4669.6 & 21409 & 1 \\
\hline 3 & 1 & 4676.2 & 21379 & 5 \\
\hline 4 & 2 & 4712.4 & 21215 & 2 \\
\hline 2 & 1 & 4722.7 & 21168 & -6 \\
\hline 5 & 3 & 4748.9 & 21052 & 0 \\
\hline 6 & 4 & 4784.8 & 21894 & 3 \\
\hline 4 & 3 & 4793.2 & 21857 & 0 \\
\hline
\end{tabular}
rations of the $A^{2} \Sigma^{+}$and $X^{2} \Pi_{1 / 2}$ states are dif-
Table 1. Bandheads for the A ${ }^{2} \Sigma^{+}-X^{2} \Pi_{1 / 2}$ emission system of SiI.

* Calculated from the following molecular constants $\left(\mathrm{cm}^{-1}\right)^{2)}: \nu_{e}=21204.9, \omega_{e}{ }^{\prime}=208.6, \omega_{e} x_{e}{ }^{\prime}=1.66$, $\omega_{e} y_{e}{ }^{\prime}=0.079, \omega_{e} z_{e}=0.0055$, $\omega_{e}^{\prime \prime}=363.8$ and (") $_{e} x_{e}^{\prime \prime}$ $=1.25$. 


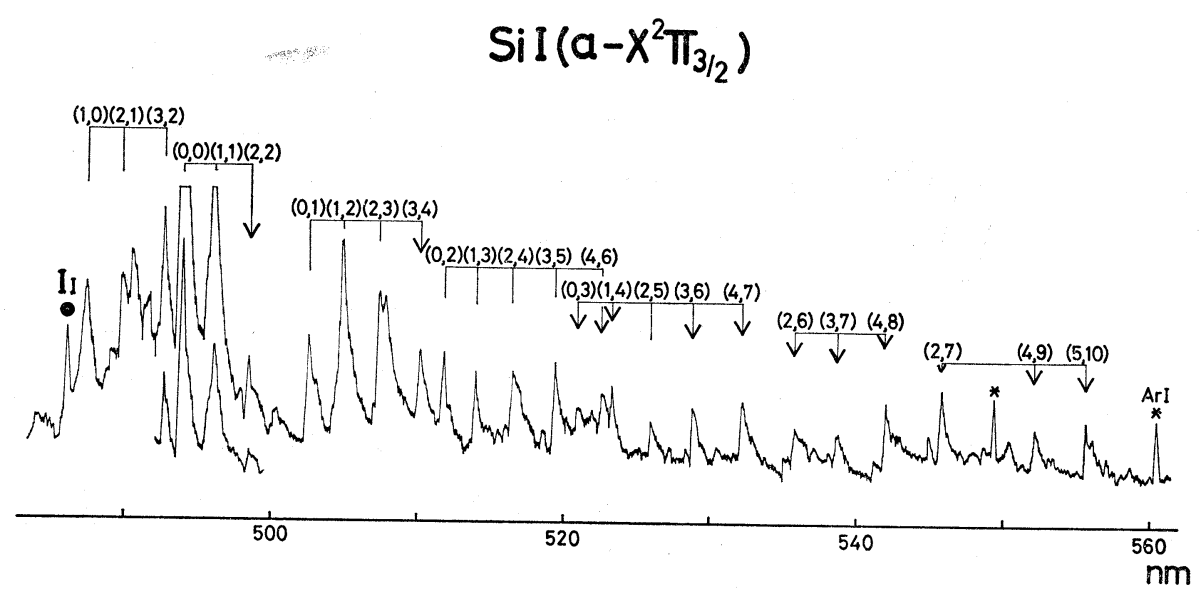

Fig. 3. The $a\left({ }^{4} \Sigma^{-}{ }_{1 / 2}\right)-X^{2} \Pi_{3 / 2}$ emission system of $\mathrm{SiI}$ in the $485-560 \mathrm{~nm}$ region.

ferent significantly.

In Fig. 3 we show an enlarged spectrum in the $485-560 \mathrm{~nm}$ region. The spectrum is composed of a number of red-degraded bands. Thirteen dominant bands extending in the $485-530 \mathrm{~nm}$ region were ascribed to the $a\left({ }^{4} \Sigma_{1 / 2}\right)$ $-X^{2} \Pi_{3 / 2}$ transition of SiI according to the assignment of Billingsley ${ }^{2}$. In this work, we could identify 13 additional bands as indicated in Fig. 3 by arrows. Their bandhead data are listed in Table 2 together with calculated

Table 2. Bandheads for the a $\left({ }^{4} \Sigma^{-}{ }_{1 / 2}\right)-X^{2} \Pi_{3 / 2}$ emission system of $\mathrm{SiI}$.

\begin{tabular}{ccccc}
\hline \hline$v^{\prime}$ & $v^{\prime \prime}$ & $\begin{array}{c}\text { Wavelength } \\
(\AA)\end{array}$ & \multicolumn{2}{c}{ Wavenumber $\left(\mathrm{cm}^{-1}\right)$} \\
\cline { 4 - 5 } & & & Observed & Cbs.-Cal.* \\
\hline 2 & 2 & 4985.1 & 20054 & 1 \\
3 & 4 & 5102.8 & 19592 & -1 \\
0 & 3 & 5211.0 & 19185 & 2 \\
4 & 6 & 5227.0 & 19126 & -4 \\
1 & 4 & 5234.3 & 19099 & 2 \\
3 & 6 & 5289.7 & 18899 & 0 \\
4 & 7 & 5323.1 & 18781 & -5 \\
2 & 6 & 5358.1 & 18658 & 1 \\
3 & 7 & 5388.2 & 18554 & -1 \\
4 & 8 & 5421.2 & 18441 & -4 \\
2 & 7 & 5459.4 & 18312 & -1 \\
4 & 9 & 5523.0 & 18101 & -5 \\
5 & 10 & 5557.9 & 17687 & -1 \\
\hline
\end{tabular}

* Calculated from the following molecular constants $\left(\mathrm{cm}^{-1}\right)^{2)}: \nu_{e}=20289.7, \omega_{e}^{\prime}=275.7, \omega_{e} x_{e}{ }^{\prime}=5,6, \omega_{e}^{\prime \prime}=$ 359.0 and $\omega_{e} x_{e}{ }^{\prime \prime}=1.1$. values. A good agreement between the observed and calculated bandheads supports our vibrational assignment. Our assignment on the $(2,2),(0,3),(1,4),(3,6)$ and $(4,7)$ bands are consistent with the numbering of Lakshminarayana and Haranath ${ }^{1)}$, though there is a discrepancy of $3-14 \mathrm{~cm}^{-1}$ between the wavenumbers of bandheads. The weak second subsystem reported by them could not be observed in this work as in the measurement of Billingsley ${ }^{2)}$. The $a\left({ }^{4} \Sigma_{1 / 2}^{-}\right)-X^{2} \Pi_{3 / 2}$ system from the lower vibrational levels, $v^{\prime}=0-3$, could be found in the He afterglow, while it was absent in the Ne afterglow.

The authors wish to express their thanks to Dr. H. Sekiya and Messers H. Obase and K. Mizukami for their helpful discussions.

\section{References}

1) A. Lakshminarayana and P.B.V. Haranath: J. Phys. B3, 576 (1970).

2) J. Billingsley: J. Mol. Spectrosc. 43, 128 (1972).

3) K. P. Huber and G. Hertzberg: Molecular Spectra and Molecular Structure, Vol. IV (Van Nostrand, New York, 1979).

4) M. Tsuji, K. Shinohara, T. Mizuguchi, and Y. Nishimura: Can. J. Phys. 61, 251 (1983).

5) D. R. Stull and H. Prophet: JANAF Thermochemical Tables, NSRDS-NBS 37 (U.S. Government Printing Office, Washington, D.C. 1971).

6) R.E. Pabst, J.L. Margrave, and J.L. Franklin: Int. J. Mass Spectrom. Ion Phys. 25, 361 (1977).

7) T. Matsuo, N. Kobayashi, and Y. Kaneko: J. Phys. Soc. Japan 51, 1558 (1982). 